\title{
Investigation of Biometric Identification Technology Based on biological Fingerprints and Facial Features
}

\author{
Huixing $\mathrm{Li}^{1,2, a}$, Yan Xue ${ }^{1,2}$ and Xiancai Zeng ${ }^{1,2}$ \\ ${ }^{1}$ Henan Key Laboratory of Industrial Microbial Resources and Fermentation Technology, Nanyang Institute of Technology, Nanyang, \\ Henan 473004, China \\ ${ }^{2}$ School of Biological and Chemical Engineering, Nanyang Institute of Technology, Nanyang, Henan 473004, China
}

\begin{abstract}
Biometric identification is largely dependent on feature extraction technology. As feature extraction techniques are increasingly mature, scholars have gradually turned their attention to the relevant problems between biometric characteristics. This paper reviews the characteristic extraction method of face and fingerprint analyzes the feature classification extraction method based on empirical knowledge and the depth learning-based computer logic sampling extraction method and compares existing solutions from the angle of image processing. Based on feature extraction, it will have prospected in the future biometric identification model of progress.
\end{abstract}

\section{Introduction}

Deep learning has an excellent performance in data analysis and accuracy and has gradually attracted the attention of various industries, and traditional biometric algorithms have also been challenged [1]. Scholars have invested in the fields of multi-biological modal identification based on machine learning/deep learning, and have achieved gratifying results in a relatively short period. It has been verified unilaterally that "the process of making machines have the ability to recognize" is an important leap for the transformation of human knowledge into human development. In contrast to the history of the development of biometric recognition, the processing of biometric images has always been the focus of scholars' research and the aid of computer equipment. This process includes the steps of extraction and analysis of relevant biological characteristics and comparison with existing data, and finally outputs the recognition results. With the introduction and application of machine learning and deep learning, the feature extraction process has received more attention. The quality of feature extraction directly affects the learning process of the model, the correctness of the model is obtained, and then the output of the entire model is affected.

Traditional identity authentication is mainly based on possessions or passwords. However, possessions such as documents are easy to lose and embezzle, and passwords are easy to forget. More importantly, in various fields that use the network as the medium, the limitations and drawbacks of traditional identity authentication methods make it unable to fully meet the requirements of electronic information security. As a result, the third method of identity authentication came into being, that is, automatic identity authentication based on a person's unique physiological or behavioral characteristics, which is now collectively referred to as biometric identification technology.

Not all biological characteristics can be used for personal identification [2]. The biological characteristics that can be used for identification must meet the following conditions: 1) Universality [3]: That is, everyone must have this characteristic. 2) Uniqueness [4]: that is, the characteristics of any two people are different. 3) Measurability [5]: the feature is measurable. 4) Stability [6]: That is, the characteristics will not change in a period. Of course, other practical factors must be considered in the application process

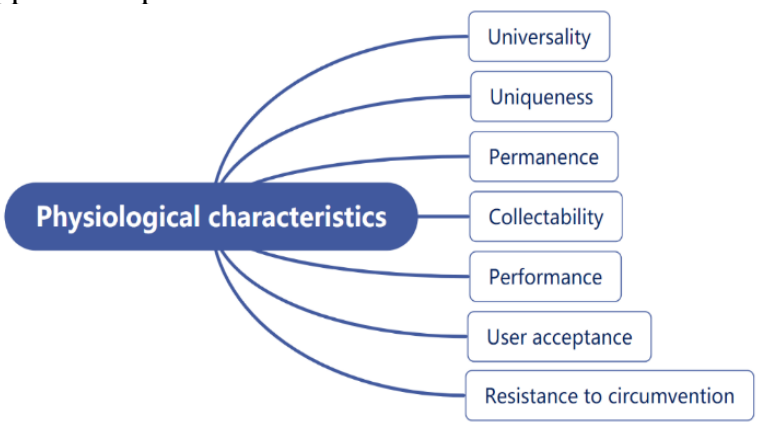

Figure 1. Characterization of physiological characteristics.

The biological characteristics that humans possess include two types of physiological characteristics and behavioral characteristics. Physiological characteristics are formed congenitally, such as DNA, fingerprints, iris, face, palm prints, etc. This kind of characteristic satisfies the basic conditions as a biological characteristic. Human beings are regarded as a species, and each person is regarded as an individual. The physiological characteristics have several characteristics as shown in Figure 1.

\footnotetext{
${ }^{a}$ Corresponding author: Lihuixing@nyist.edu.cn
} 
Therefore, biometrics is an excellent choice for identity verification. Behavioral characteristics are formed with acquired habits, including voice, handwriting, gait, and vein structure. Such characteristics are stable over a while but have the potential to change over time.

The feature extraction of biological images is one of the important steps in the biometric recognition system, and it is of great significance to the entire biometric recognition process: The current biometric recognition system mostly uses the method to determine the biological characteristics to be identified through feature comparison and the number of feature matching pairs Consistency with the biological characteristics of the sample. Therefore, the completeness and accuracy of feature extraction are directly related to the recognition accuracy and robustness of the biometric system.

\section{Fingerprint image feature extraction method}

Fingerprint image feature extraction is based on the division and preprocessing operations of fingerprint image regions, as well as the prior conclusions about fingerprint features of human beings so far-the knowledge of fingerprint features themselves. This paper reviews two aspects of traditional feature extraction methods and feature extraction methods based on deep learning.

Traditional fingerprint feature extraction methods analyze the image characteristics of existing fingerprint images (including gray-scale features, texture features, frequency characteristics, etc.), and combine the prior knowledge of biological characteristics (sometimes supplemented by the mathematical and logical expression of the prior knowledge in disguised form). It is solidified in the feature extraction algorithm in the form of rules to complete the feature extraction task. According to the goal of the extraction task, traditional fingerprint feature extraction methods can be divided into the following two categories. The first category is based on the calculation of the direction of the fingerprint image, which is to complete the estimation and judgment of the local direction field or the overall direction field of the fingerprint. The second type of method uses the graphic knowledge of fingerprint images as a template to solidify into the image feature recognition system to extract features or derive based on the geometric characteristics of the image, and use mathematical tools to change the data needed in the process of extracting features to extract features.

The first type of method can be roughly subdivided into three types: the method based on the gradient vector, the method based on the filter, and the method based on the model. The gradient vector-based method calculates the gradient vector of the fingerprint image at each pixel point and takes the vertical direction of the fastest gradient change direction of the center point in the specified subregion as the ridge direction of the fingerprint image at that point. This method requires high noise removal ability in the preprocessing step and has poor robustness. The noise in the image can easily interfere with the determination of the ridge direction. The filtering-based method also requires a higher noise removal effect when calculating the direction, but the effect is better in the local area. The model-based method considers the global constraints and distribution rules of the fingerprint orientation field except for the area near the fingerprint singular points.

The second type of feature extraction method is mainly for the characteristics of the fingerprint pattern area. The more typical methods are the method of locating singular points by counting the changes in the direction of the subregion on the closed curve around the sub-region of the image; in the direction map of the partial area of the fingerprint A semicircular area containing 3 parts (including the center point) is defined, and the method of locating the center point by analyzing the magnitude relationship of the sine component of the direction in the area; using the innermost layer of the fingerprint to have no minutiae feature points in a fixed step The method of determining the singularity point by analyzing the directional consistency of the image sub-blocks around the image sub-block in the block direction map by analyzing the vertices of the arc (the place with the largest curvature on the ridge) as the center point. The smaller the area, the better, but pay attention to the moderate selection of the area, otherwise it will reduce the anti-noise performance of the algorithm. Besides, researchers have explored the mathematical properties of fingerprint image features and applied them to the process of extracting features, and the results obtained are also very significant: For example, the singular point extraction is completed by constructing two different types of symmetric filters. The method is to transform the fingerprint extraction into the extraction of the statistical data of the image itself under certain restricted conditions in a disguised manner through the filtering operation. This method is used to extract the largest circular symmetry point and the triangle symmetry point in the fingerprint image and use it as a singular point. Determine the location of the singular point can determine the direction at the same time, but it is affected by image noise/edge effects. On this basis, an algorithm for extracting singular points from the enhanced image is proposed, which improves the defect that the original process is susceptible to noise and edge effects.

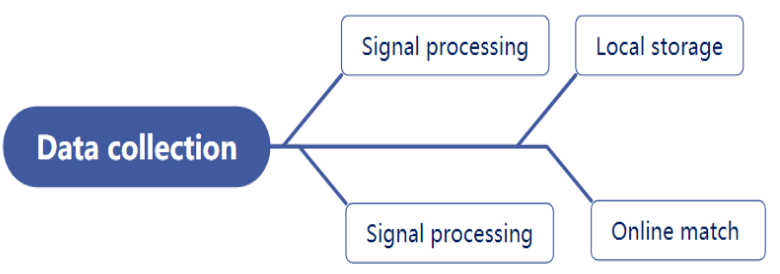

Figure 2. Biometric system and subsystem framework.

\section{Face feature extraction method based on face representation}

According to different face representation data, face representation can be divided into 2D-based face representation methods and $3 \mathrm{D}$-based face representation methods. After the characterization, the compact feature vector obtained by the face image conversion and can be used for discrimination is obtained. In traditional face 
recognition algorithms, the extraction of facial features in images relies on features summarized by experience (prior knowledge, such as edge, texture description, etc.) and classic machine learning methods (principal component analysis, linear discriminant analysis, support vector machine, etc.). The characteristic criterion obtained from the empirical summary is less robust when dealing with changes in external conditions or large changes in the individual's characteristics in an unconstrained environment, which makes the past researchers focus on prescribing the right medicine, such as different ages., Different postures and different lighting conditions.

As AlexNet shines in ImageNet, deep learning models represented by a convolutional neural network (CNN) have gradually replaced traditional face recognition methods. Facial feature extraction is also changed from the inherent patterns in the traditional biometric recognition process and the feature classification extraction based on face knowledge to the deep feature mining and extraction process based on computer logic. The main advantage of the deep learning method is that its learning process is carried out on a data set with a large number of basic facial features, and the optimal feature expression mode of these data is learned according to the set objective function. A large number of natural face images available on the Internet has allowed researchers to collect large-scale face data sets. These images contain various possible changes in the expression of human faces in the real world. The $\mathrm{CNN}$-based face recognition method trained with these data sets has reached a very high accuracy rate because the model itself can learn the robust feature expression in the face image, to be able to cope with the face image used in the training process The changes in the real world presented.

The current CNN model used in face recognition can be trained using different methods according to the target data cluster and the ultimate goal to be achieved. Among them, the problem is regarded as a classification problem, and each subject in the training set corresponds to a category is a more common method. In the CNN model after training, the classification layer is removed, the features of the previous layer are used for face characterization, and the model is used to identify targets that do not exist in the training set. In the related research of deep learning, these features are usually called bottleneck features. After this training stage, the model can still be further trained using other techniques, and use this as a goal to apply optimization.

\section{Conjecture about the correlation between the face and fingerprint features}

Face features and fingerprint features are essentially the expression results of two completely different biological traits, but based on the current findings-face features can be used as a powerful means of identity detection, and fingerprint features are also very different. Biological characteristics, both of which have been maturely applied to the identity verification system of all walks of life. In an era when machine learning and deep learning are booming, applications based on multi-modal biometrics and gender verification algorithms have also been proposed and gradually improved. Therefore, it cannot be said that there is no correlation between the two. But this kind of correlation is really beyond the scope of explanation of the current scientific level of mankind. Therefore, it is hoped to think about how to explore the correlation between fingerprints and human faces based on the multi-modal feature layer fusion process. The basis for the realization of this idea has the following 3 points:

1) The feature processing process of biological images is based on the classification process (whether it is a machine vision model or a person's visual system), and the limit of human vision acceptance is limited by human cognition, even if the person has enough knowledge The time required to transform it into a model application far exceeds that of a computer, so humans can use computers as an auxiliary tool to speed up the rate of acquiring knowledge.

2) With the transformative impact of deep learning on image processing, machine vision has surpassed human vision in cognitive level and recognition ability. Although the current recognition ability is only limited to subdisciplines such as identity verification, there are existing the deep learning model is firmly advancing along the theories left by neuroscience. Human bioelectric signals ( $+40 \mathrm{mV}$ and $-70 \mathrm{mV}$ ) and the computer's $0 / 1$ input, the synaptic receptors between human nerve cells, are advancing firmly. Growth and the training and parameter adjustment process in machine learning/deep learning, etc., are gradually verifying humans' conjectures about the nature of learning, biological intelligence generated from natural evolution. Therefore, the conversion between biological characteristics is realized by establishing a multi-layer transfer model. This proposition is a reasonable conjecture.

3) The process of DNA transcription + RNA translation is used to build a correlation model between facial features and fingerprint features. This model is not just limited to the two. This is an extensible model framework to be explored. , It can cover the current popular gait, voice, and other features within the scope of biometric recognition. Its meaning is similar to DNA, but the process of obtaining is transformed from inside the organism (that is, extracting DNA) to Obtain from information carriers such as images, videos, and audios. Based on this, there is a practical conjecture about the existence of a certain framework that can be converted between biological characteristics.

\section{Conclusion}

By reviewing the relevant progress of fingerprint feature extraction, face feature extraction, and multi-modal biometric recognition, this paper summarizes the biometric feature extraction methods from the perspective of feature extraction and compares the feature extraction methods of face and fingerprint in depth. The limitations of the current multi-modal biometric recognition and single-modal biometric recognition are analyzed; based on the problem of unifying the expression of different 
biometrics faced by the fusion of the feature layer, we propose whether the features of human face and fingerprint. There are problems of relevance, and it extends to the evaluation methods and model ideas under the condition of the correlation of human faces and fingerprints. The conjecture of constructing a unified biological image feature expression model is proposed.

\section{Acknowledgments}

This research work is supported by the Natural Science Foundation of Henan Province of China (182300410151); Scientific and Technological Project of Henan Province (162102210114); Key Scientific Research Project of College and University in Henan Province (17A610003); Henan Key Laboratory of Industrial Microbial Resources and Fermentation Technology Open Project (HIMFT20200206); Interdisciplinary Sciences Project, Nanyang Institute of Technology.

\section{References}

1. Alay, N., \& Al-Baity, H. H. . (2020). Deep learning approach for multimodal biometric recognition system based on fusion of iris, face, and finger vein traits. Sensors, 20(19).

2. Ramenzoni, L. L. , \& Line, S. R. P. . (2006). Automated biometrics-based personal identification of the hunter-schreger bands of dental enamel. Proceedings of the Royal Society B Biological Sciences, 273(1590), 1155-1158.

3. Braun, E. , \& Marom, S. . (2015). Universality, complexity and the praxis of biology: two case studies. Studies in History \& Philosophy of Biological \& Biomedical Sciences, 53, 68-72.

4. Mehrabian, A. . (1992). Interrelationships among name desirability, name uniqueness, emotion characteristics connoted by names, and temperament. Journal of Applied Social Psychology, 22(23), 17971808.

5. Gajda, J. . (1995). Analysis of measurability of complex identification objects. Systems Analysis Modelling Simulation(3).

6. Yi, M., Min, M., Yun, D. , \& An, H. . (2010). Expression, identification and biological effects of a novel vpac2-specific agonist with high stability and bioactivity. Acta Biochim Biophys Sin(1), 21-29. 